\title{
A Prospective Study of the Effect of Tinnitus Sound Matching Degree on the Efficacy of Customized Sound Therapy in Patients with Chronic Tinnitus
}

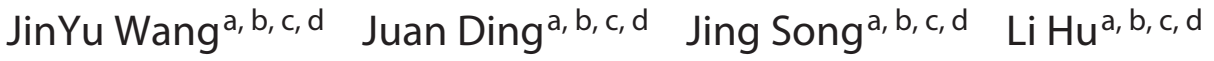 \\ Ning Cong a, b, c, d Zhao Han d, e
}

\begin{abstract}
aDepartment of Otology and Skull Base Surgery, Eye Ear Nose \& Throat Hospital, Fudan University, Shanghai, China; bShanghai Auditory Medical Center, Shanghai, China; 'NHC Key Laboratory of Hearing Medicine (Fudan University), Shanghai, China; ${ }^{d}$ Fudan University, Shanghai, China; ${ }^{~}$ Department of Otorhinolaryngology, HuaDong Hospital Affiliated to Fudan University, Shanghai, PR China
\end{abstract}

\section{Keywords}

Chronic tinnitus · Tinnitus sound matching · Customized sound therapy · Sound therapy effect

\begin{abstract}
Objectives: The aim of this study was to explore and compare the customized sound therapy effect between tinnitus sound matching and nonmatching patients in tinnitus customized sound therapy and therapy-related influencing factors. Methods: This prospective study investigated a total of 100 patients with unilateral chronic tinnitus who received customized sound therapy. The participants were dichotomously divided into matching (group A) and nonmatching (group B) groups after 4 stages of tinnitus matching via the tinnitus assistant app (provided by Sound Ocean Company, SuZhou, China). Each group consists of 50 participants. Before and 6 months after the treatment, Hospital Anxiety and Depression Scale (HADS), tinnitus handicap inventory (THI), and tinnitus loudness Visual Analog Scale (VAS) were used to evaluate the customized sound therapy effect and explore
\end{abstract}

other related influencing factors. Results: (1) The HADS-A, HADS-D, THI, and VAS scores of 2 groups were both significantly decreased after treatment. (2) The HADS-A and THI scores improved markedly in group $A$ than that in group $B$, which could be related to the hearing loss of the tinnitus side ear before treatment; the lighter the degree of hearing loss, the better the improvement. No statistically significant differences were detected in HADS-D and VAS scores between the 2 groups, and also, these were not related to the degree of hearing loss. The differences in age, gender, and tinnitus duration did not show any statistically significant effect on the improvement of the 2 groups. Conclusions: Both tinnitus sound matching and nonmatching of the customized sound therapy brought a significant effect to tinnitus participants. Our study also suggests that THI and HADS-A scores of those with tinnitus matching participants improved markedly as compared to those of nonmatching participants, and the customized sound therapy effect is negatively correlated with the severity of hearing loss.

(C) 2021 The Author(s)

Published by S. Karger AG, Basel
(C) 2021 The Author(s)

Published by S. Karger AG, Basel

This is an Open Access article licensed under the Creative Commons Attribution-NonCommercial-4.0 International License (CC BY-NC) (http://www.karger.com/Services/OpenAccessLicense), applicable to the online version of the article only. Usage and distribution for commercial purposes requires written permission.
Correspondence to:

Ning Cong, congningent@126.com

Zhao Han, sfhanzao@163.com 


\section{Introduction}

Tinnitus is one of the most common chief complaints in the otorhinolaryngology clinic. It is often divided into subjective and objective tinnitus. Hitherto, the mechanism of subjective tinnitus is not completely elucidated. Based on the results of current research, it is hypothesized that subjective tinnitus originates in the peripheral auditory system and is developed and maintained in the central system due to central remodeling, which is the result of adaptive changes causing auditory cortex abnormalities secondary to hearing impairment [1]. The subjective tinnitus had different treatment methods. The commonly used clinical symptomatic treatments include drug treatment, sound therapy, tinnitus retraining therapy (TRT), psychological treatment [1], and tinnitus activities treatment [2].

In recent years, based on the principle of central remodeling, Okamoto et al. [3] proposed the use of customized sound to personalize the treatment of subjective tinnitus, which reduces tinnitus-related cortical activity and subjective tinnitus sensation. Previous studies have shown that sound therapy effectively reduces the excitability of tinnitus brain neural activity [4]. With the development of clinical diagnosis and treatment, the clinical efficacy of customized sound for tinnitus has been affirmed, and there are almost no side effects rendering it as a favorable method [3].

Hospital Anxiety and Depression Scale (HADS), tinnitus handicap inventory (THI), and tinnitus loudness Visual Analog Scale (VAS) are used to describe the severity of tinnitus and evaluate of tinnitus curative effect. According to our clinical experience, the 3 questionnaires THI, HADS, and VAS have demonstrated their internal consistency, using the 3 aforementioned scales at the same time helped us to comprehensively evaluate the efficacy of tinnitus sound therapy. HADS is composed of 7 anxiety scoring items (HADS-A) and 7 depression scoring items (HADS-D), each with a score of $0-21$; the higher the score, the more serious the anxiety or depression symptoms. It is widely used to score the severity of anxiety and depression in tinnitus patients [5], THI is based on patient's subjective feelings of tinnitus perception, include 25 items grouped into functional, emotional, and catastrophic subscales, which can be used to quantify the impact of tinnitus in daily life. And the scores ranged from 0 to 100 points according to the degree of tinnitus impact on the patients. The higher the patient's score, the more severe the effect of tinnitus; it is used to show the degree of the negative effect of tinnitus [6]. VAS is a vi- sual scale used to mark the loudness of patient's tinnitus on scale from 0 to 10 points, and the loudness of tinnitus is subjectively judged by the patient. The higher the score, the higher the tinnitus loudness, that is, more serious the impact of tinnitus to patients.

While customizing personalized therapeutic sounds, the tinnitus sound matching is oftentimes considered as a critical reference factor [7]. However, we found that the customized sound could not match the tinnitus sound of some patients during sound therapy, and the customized sound therapy effect of these patients remains inconclusive. Tinnitus matching commonly includes loudness matching and pitch matching, and specifically, Pan found that results of tinnitus pitch matching were inconsistent with their primary frequency of hearing loss [8]. Furthermore, a study on the differential effects of different tinnitus sound matching degrees with respect to the curative effect of chronic subjective tinnitus by customized sound therapy is yet lacking. Therefore, this prospective study enrolled 100 patients who received customized sound therapy with chronic subjective tinnitus. The patients were classified as those with matching tinnitus sounds and those with nonmatching tinnitus sounds and compared to explore the curative effect; also, other related factors that may affect these results were investigated.

\section{Materials and Methods}

\section{Clinical Data}

A total of 100 patients with tinnitus as the first complaint were enrolled and treated in the Otology Clinic of the Eye, Ear, Nose, and Throat Hospital of Fudan University from December 2017 to June 2019. The inclusion criteria included age $\geq 18$ years and unilateral chronic tinnitus for $\geq 6$ months with normal middle ear function, with or without sensor neural hearing loss. Pure-tone average (PTA) of the tinnitus side ear was used to estimate patient's hearing, classified as normal (if PTA was -10 to $25 \mathrm{~dB} \mathrm{HL}$ ) and hearing loss (if PTA was $>25 \mathrm{~dB}$ HL, the degree worsen as PTA increased). The exclusion criteria included patients with ear disease, such as otitis media, acoustic neuroma, and objective tinnitus, such as middle ear myoclonus and vascular pulsatile tinnitus, and systemic diseases that may cause tinnitus symptoms, such as hyperthyroidism, high blood pressure, and diabetes, poor compliance, and communication problems, and those currently under other tinnitus treatments. The exit criteria included patients who chose to quit customized sound therapy according to their own considerations and those who no longer met the inclusion criteria, for example, abnormal vital organ function, poor compliance, aggravation of the disease, serious adverse reactions that require stopping treatment and adopting other treatment methods, or subjects who chose not to quit voluntarily. More than 300 patients were enrolled to this study and 200 patients have been excluded. The patients who received the sound therapy were informed and well-educated prior to the execution. All the subjects (or their par- 
Table 1. Characteristics of each treatment group

\begin{tabular}{lll}
\hline Characteristic & Group A & Group B \\
\hline Numbers & 50 & 50 \\
Matching degree & Match & Nonmatch \\
Average age (years) & $43.04 \pm 11.13$ & $43.44 \pm 12.76$ \\
Sex (male/female) & $25 / 25$ & $27 / 23$ \\
Average hearing of tinnitus side ear (DB HL) & $2.94 \pm 2.82$ & $3.00 \pm 2.89$ \\
\hline
\end{tabular}

ents or guardians) have given their informed consent and signed their consent on the outpatient case file before treatment.

In the pre-experiment, 5 patients were randomly assigned to each of the 2 groups, and the sample size was calculated according to the results of the pre-experiment. The two-population mean hypothesis test with a completely random design was adopted. The test level was $\alpha=0.05$ and $\beta=0.2$, and the ratio of sample size between the 2 groups was 1:1. The two-sided test was conducted without considering the loss of follow-up. The sample size was estimated by the difference between the first follow-up score and the baseline score and the standard deviation of the difference between the 3 subjective evaluation scales mentioned in the text. The maximum sample size calculated by the 3 scales was set as the required sample size for this study ( 45 cases). In this study, 50 patients were included in both groups as the sample size.

The cohort comprised 37 male and 63 female patients. The average age was $43.24 \pm 11.76$ (range: $18-80$ ) years, the average tinnitus duration was $2.97 \pm 2.84$ years (range: 6 months -10 years), and the average hearing of tinnitus side ear was $28.18 \pm 14.53$ (range: 6-68) dB HL. According to the tinnitus sound matching results, the patients were dichotomously divided into matching (group A) and nonmatching (group B) group. All patients underwent routine physical examination and pure-tone hearing threshold test and filled out the tinnitus evaluation survey. If necessary, imaging examination was performed to exclude the organic lesions of the ear. Both matching group included 50 patients and the nonmatch group included 50 patients.

\section{Tinnitus Sound Matching and Grouping Method}

Each patient underwent the tinnitus sound matching test on the tinnitus assistant app (provided by Sound Ocean Company, SuZhou, China) using the EdifierW800BT headphone. The test was performed in a quiet environment, and all procedures were carefully educated by the professional instructors. The first step was to classify the characteristics of patient-specific tinnitus in 3 broad categories, that is, common tinnitus sounds, electronic sounds, and normal daily life sounds. These 3 tinnitus sound categories were based on the wide range of sounds experienced by tinnitus sufferers which were first documented by Tyler and Baker [9]. Dauman and Tyler attempted to clearly distinguish the mechanism of tinnitus from the reactions to tinnitus [10]. The second step was to carry out the second-level classification of the initial 3 categories of tinnitus sounds. The common tinnitus sounds include tonal/ringing, hissing/buzzing sound, or insect sound; the electronic sounds include different types of motor/mechanic sounds; and the normal daily life sounds include television snowflake sounds and kettle boiling sounds. The third step was to match patient-specific frequency classification according to the previously chosen tinnitus type. The last step was to match the patient's tinnitus loudness according to the different levels of volume from degree 1 to 10 which is settled by the procedure, the higher the numerical scores, the louder the tinnitus sound. After finishing all 4 steps, these patients whose tinnitus sounds matched the given simulated sounds were categorized as group A. If one's tinnitus sound did not match the given simulated tinnitus sounds in the application, then the person's tinnitus sound was uploaded to the application according to the person's descriptions; these patients comprised group B. Characteristics of each treatment group have been shown in Table 1.

\section{Sound Therapy Protocols}

The patient-specific tinnitus evaluation test including tinnitus sound matching test results, audiology test, and various tinnitus impact evaluation forms were input into the tinnitus assistant app's core patent software (software simulating tinnitus-related brain), to simulate the central response of tinnitus and capture the changes in the brain's auditory system, considering that patients in group B do not match the tinnitus sound matching test, the parameter setting of group B was composed of the patient's subjective tinnitus sound description, audiology test and various tinnitus impact evaluation forms. According to each patient's personalized parameters, the software applies the specific algorithm to edit the sound materials and customizes the personalized sound suitable for the patient to eliminate or modulate the changes in the auditory system of tinnitus patients. The whole procedure was performed using the customized personalized tinnitus sound method (strategies provided by Sound Ocean Company and approved by the US FDA), the refined customized tinnitus sounds for different patients are charged. Meanwhile, the follow-up and counseling systems have been installed in the paid section under the education of professionals; improprieties in the treatment process would be pointed out and corrected in time.

The patients were instructed to use recommended headphones to receive personalized treatment every day in a quiet environment without external sound interference. The cumulative time of listening to customized music was a minimal of $2 \mathrm{~h} / \mathrm{day}$, and the treatment implementation of each patients include the treatment time and duration was automatically uploaded to the Cloud in time and recorded by professionals in this software. The loudness of therapeutic sound was similar to the tinnitus loudness, to which degree patient's tinnitus sound can also be heard when listening carefully to therapeutic music. All patients were followed up after 6 months of treatment through the tinnitus assistant app. Electronic tinnitus assessment instruments were given and back-andforth communications about treatment-related problems were replied. We will follow-up the patients regularly by phone or email through the patented software. Meanwhile, patients will be followed up monthly in outpatient clinics, to ensure the accuracy of patient data. The flow diagram is shown in Figure 1. 
Fig. 1. Flow diagram of this study.

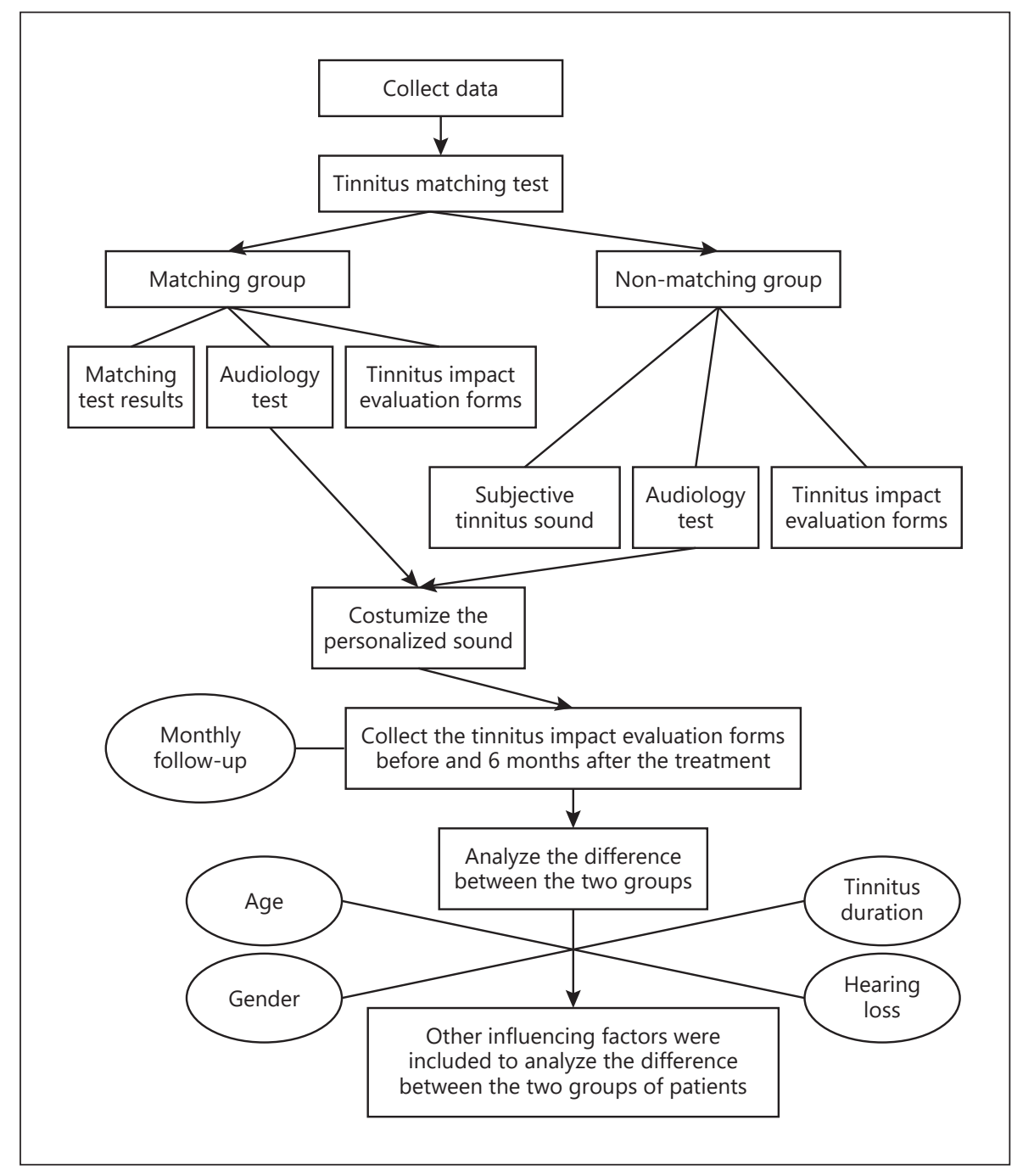

Evaluation Methods of the Tinnitus Curative Effect

Our previous study showed that patients' tinnitus changed in the same trend after 1, 3, and 6 months of the beginning of the tinnitus treatment; based on this, we chose to take the longer time (6 months after treatment) as the study standard [11]. Before and 6 months after the treatment, all patients in either group filled out the HADS, THI, and VAS distributed on the tinnitus assistant app inventory. The numerical data were collected by professional instructors. We compared the changes in HADS-A, HADS-D, THI, and VAS scores before and 6 months after the treatment to evaluate the curative effect of each group. In addition, the gender, age, tinnitus duration, and hearing loss before the treatment were included to analyze the difference between the 2 groups of patients.

\section{Statistical Analysis}

To investigate whether the significant difference exists in the hearing characteristics between patients before and after treatment, the differences in the 4 indexes of HADS-A, HADS-D, THI, and VAS before and after treatment were analyzed in patients. The paired sample $T$ test method was used to compare numerical data before and after treatment. A multiple linear regression model was selected to fit previously considered confounders of the symptoms related to tinnitus and patients' characteristics to investigate a significant difference between the 2 group using entry methods. All remaining variables were adjusted when evaluating the relationship between the posttreatment scores and other variables. $p<0.05$ is considered statistically significant. All statistical analyses were performed using SPSS 21.0 software (SPSS Inc., Chicago, IL, USA).

\section{Results}

\section{Curative Effect of Sound Therapy in the Two Groups}

Table 2 demonstrates that the mean HADS-A score decreased from $10.6 \pm 3.93$ to $5.24 \pm 4.06(p<0.001)$, the mean HADS-D score decreased from $9.02 \pm 4.08$ to 4.38 $\pm 3.85(p<0.001)$, the mean THI score decreased from 
Table 2. Indexes of group A before and after treatment $(n=50)$

\begin{tabular}{lccc}
\hline Index & $\begin{array}{l}\text { Pretreatment } \\
\text { scores }\end{array}$ & $\begin{array}{l}\text { Posttreatment } \\
\text { scores }\end{array}$ & $p$ value \\
\hline HADS-A & $10.60 \pm 3.93$ & $5.24 \pm 4.06$ & $<0.001$ \\
HADS-D & $9.02 \pm 4.08$ & $4.38 \pm 3.85$ & $<0.001$ \\
THI & $59.68 \pm 23.95$ & $33.72 \pm 23.95$ & $<0.001$ \\
VAS & $6.10 \pm 2.59$ & $3.26 \pm 2.17$ & $<0.001$ \\
\hline
\end{tabular}

The variables are expressed as mean \pm SD. HADS, Hospital Anxiety and Depression Scale; THI, tinnitus handicap inventory; VAS, Visual Analog Scale; SD, standard deviation.

$59.68 \pm 23.95$ to $33.72 \pm 23.95(p<0.001)$, and the mean VAS score decreased from $6.10 \pm 2.59$ to $3.26 \pm 2.17$ ( $p<$ 0.001 ) in group A. It showed that the scores of HADS-A, HADS-D, THI, and VAS after receiving tinnitus sound matching sound therapy were significantly lower than before the treatment.

Table 3 exhibits that the mean HADS-A score decreased from $10.64 \pm 3.96$ to $6.30 \pm 4.70(p=0.013)$, the mean HADS-D score decreased from $8.62 \pm 3.48$ to 4.96 $\pm 4.49(p=0.024)$, the mean THI score decreased from $54.68 \pm 22.60$ to $31.16 \pm 24.99(p<0.001)$, and the mean VAS scores decreased from $5.86 \pm 2.18$ to $3.44 \pm 2.25$ $(p<0.001)$ in group B. It showed that the scores of HADS-A, HADS-D, THI, and VAS after receiving tinnitus sound nonmatching sound therapy were significantly lower than before the treatment.

\section{Results of the Multiple Linear Regression Analysis of} the Influencing Factors and Curative Effect Differences between the 2 Groups

From the first regression results in Tables 4 and 5, the regression coefficient of the core dependent variable tinnitus sound matching degree is $-2.177(p=0.008)$. From the third regression results in Tables 4 and 5 , the regression coefficient of the core dependent variable is -9.969 ( $p=0.017)$, indicating that the HADS-A and THI scores after receiving matching sound therapy were significantly lower than those before receiving nonmatching sound therapy. Thus, it could be deduced that receiving customized matching sound therapy is better than receiving customized nonmatching sound therapy in order to improve the HADS-A and THI scores. Multivariate results showed that the degree of hearing loss is negatively correlated with the decline in the scores of HADS-A and THI indexes. The other control variables did not exert any significant effect on the HADS-A and THI indexes after treatment.

Relation of Matching Degree and Therapeutic Assessment in Tinnitus
Table 3. Indexes of group B before and after treatment $(n=50)$

\begin{tabular}{lccr}
\hline Index & $\begin{array}{l}\text { Pretreatment } \\
\text { scores }\end{array}$ & $\begin{array}{l}\text { Posttreatment } \\
\text { scores }\end{array}$ & $p$ value \\
\hline HADS-A & $10.64 \pm 3.96$ & $6.30 \pm 4.70$ & 0.013 \\
HADS-D & $8.62 \pm 3.48$ & $4.96 \pm 4.49$ & 0.024 \\
THI & $54.68 \pm 22.60$ & $31.16 \pm 24.99$ & $<0.001$ \\
VAS & $5.86 \pm 2.18$ & $3.44 \pm 2.25$ & $<0.001$ \\
\hline
\end{tabular}

The variables are expressed as mean \pm SD. HADS, Hospital Anxiety and Depression Scale; THI, tinnitus handicap inventory; VAS, Visual Analog Scale; SD, standard deviation

Table 4. Regression results of the correlation between tinnitus sound matching degree and curative effect

\begin{tabular}{llll}
\hline Index & $\begin{array}{l}\text { Regression } \\
\text { coefficient }\end{array}$ & SE & $p$ value \\
\hline HADS-A scores posttreatment & -2.177 & 0.803 & 0.008 \\
HADS-D scores posttreatment & -0.899 & 0.799 & 0.263 \\
THI scores posttreatment & -9.969 & 4.111 & 0.017 \\
VAS scores posttreatment & 0.576 & 0.425 & 0.178 \\
\hline
\end{tabular}

HADS, Hospital Anxiety and Depression Scale; THI, tinnitus handicap inventory; VAS, Visual Analog Scale; SD, standard deviation; $\mathrm{SE}$, standard error.

From the second regression results in Tables 4 and 5, the regression coefficient of the core dependent variable is 0.899 ( $p=0.263)$. From the fourth regression results in Tables 4 and 5, the regression coefficient of the core dependent variable is $0.576(p=0.178)$, indicating that there is no significant difference between the HADS-D and VAS scores after receiving sound matching and nonmatching therapy. The other control variables did not have any significant effect on the HADS-D and VAS scores after the treatment.

\section{Discussion}

Tinnitus is one of the most common symptoms that can only be perceived by the patients subjectively. Tyler focused on the 4 primary activities impaired by tinnitus often occurred in emotions, hearing, sleeping, and concentration [12]. It affects the patient's daily life, affects concentration and sleep quality so that hinders mental work, causes emotional diseases, such as anxiety and depression, and, to 
Table 5. Regression results of the correlation between influencing factors and curative effect

\begin{tabular}{|c|c|c|c|c|c|c|c|c|}
\hline & \multicolumn{2}{|c|}{$\begin{array}{l}\text { HADS-A scores } \\
\text { posttreatment }(n=100)\end{array}$} & \multicolumn{2}{|c|}{$\begin{array}{l}\text { HADS-D scores } \\
\text { posttreatment }(n=100)\end{array}$} & \multicolumn{2}{|c|}{$\begin{array}{l}\text { THI scores } \\
\text { posttreatment }(n=100)\end{array}$} & \multicolumn{2}{|c|}{$\begin{array}{l}\text { VAS scores } \\
\text { posttreatment }(n=100)\end{array}$} \\
\hline & $B$ & SE & $B$ & SE & $B$ & SE & $B$ & SE \\
\hline \multicolumn{9}{|l|}{ Core dependent variable } \\
\hline Tinnitus matching degree & $-2.177^{* * *}$ & 0.803 & -0.899 & 0.799 & $-9.969^{*}$ & 4.111 & 0.576 & 0.425 \\
\hline \multicolumn{9}{|l|}{ Control variables } \\
\hline Age, years & -0.016 & 0.035 & 0.008 & 0.035 & 0.005 & 0.183 & 0.008 & 0.019 \\
\hline Tinnitus duration & 0.209 & 0.147 & 0.195 & 0.148 & 0.975 & 0.778 & 0.043 & 0.081 \\
\hline Degree of hearing loss & $-0.079 *$ & 0.038 & 0.071 & 0.037 & $-0.513^{* *}$ & 0.192 & 0.013 & 0.020 \\
\hline
\end{tabular}

HADS, Hospital Anxiety and Depression Scale; THI, tinnitus handicap inventory; VAS, Visual Analog Scale; SD, standard deviation; SE, standard error. ${ }^{* * *} p<0.001 .{ }^{* *} p<0.01 .{ }^{*} p<0.05$.

an extreme, makes some individuals prone to suicide. Tinnitus is at the same time an intractable symptom that plagues many clinicians. The difficulty of diagnosis and treatment is ascribed to subjective tinnitus patients who do not have any detectable signs of disease, and hence, there is almost no objective technology to display the existence of tinnitus. Subjective tinnitus has many forms of sounds, which can persist or only appear temporarily. Typically, it is impossible to correlate the occurrence of tinnitus with a specific time or event [1]. In most cases, the possible cause of tinnitus is abnormal excitability at the level of the brainstem, subcortex, or cerebral cortex caused by damage to the peripheral auditory system [13].

Tyler proposed the psychological model which suggested that the overall annoyance of the tinnitus was a result of the tinnitus characteristics and the psychological makeup of each individual patient. Several parts of the brain will be involved in the representation of the tinnitus and of the reactions to the tinnitus. Treatments can be focused on reducing the tinnitus (e.g., pills) or on reducing the reactions to the tinnitus (e.g., counseling) [14]. Hitherto, the commonly used clinical treatment methods for subjective tinnitus include tinnitus masking therapy and TRT. Interestingly, the long-term clinical practice has deemed these 2 methods as effective therapeutic methods for patients; Nonetheless, TRT has been considered a better treatment method in contrast to the tinnitus masking therapy, and hence, TRT has gained a broader clinical application [15]. Meanwhile, Tyler found that TRT is not better than total masking, since the mixing point is too high for most patients, and might increase tinnitus or increase hearing loss or interfere with speech perception
[16]. And they proposed another effective therapy method, tinnitus activities treatment, which concentrates on the 4 primary functions affected by tinnitus: (1) thoughts and emotions, (2) hearing, (3) sleep, and (4) concentration [2]. In recent years, sound therapy has been used in the clinic as one of the main portions of TRT. It has been clinically evaluated as the first choice for chronic subjective tinnitus [17]. A recent study, without any counseling, has shown that sound therapy on its own, can help some tinnitus sufferers [18]. Li et al. [19] investigated the frequency-based personalized music therapy through randomized controlled trials and found that the THI score was decreased significantly, affirming its effectiveness for tinnitus treatment. Mahboubi et al. [20] found that customized sound therapy can be performed based on the Web, which helps overcome the limitations of traditional tinnitus masking therapy. It is reported that tinnitus loudness and annoyance decreased markedly after listening to the customized sound for $1 \mathrm{~h}$ [20]. The sound therapy method used in the current study was a personalized Webbased customized treatment for various characteristics of chronic tinnitus in different patients. It is a comprehensive treatment method certified, rather than a single TRT treatment method. In addition to the soothing effect of TRT on tinnitus, it is customized for different types of tinnitus of patients. Before receiving treatment, patients in both groups would receive the same education guide as they should understand the principle of tinnitus and treatment methods. After perfect communication, patients would choose whether to receive sound therapy or not according to their voluntary wishes. According to the tinnitus sound matching and not audiological characteristics, 
symptoms of tinnitus patients are submitted to patented software with artificial intelligence to simulate tinnitusrelated brain, identify the excitement parts of the brain and tinnitus-related lesions, and perform corresponding fixed-point editing processing on the music frequency through the computer model. Patients gradually reduce the activity of brain remodeling neurons by listening to customized sounds to alleviate tinnitus [21]. Moreover, it was also shown that the customized sound therapy reduces the THI scores of tinnitus patients and ameliorates the negative effects and distress caused by chronic tinnitus, while the curative effect is not affected by gender, tinnitus type, and the presence or absence of hyperacusis [11].

Clinically, tinnitus matching is often used as a critical indicator of tinnitus measurement method and tinnitus treatment evaluation [22]. Some researchers proposed that personalized sound therapy is significantly related to tinnitus pitch and loudness matching test results [23]. In our study, evaluation of the tinnitus sound matching degree served as an indicator for patients need to be tested before customizing the music. As our tinnitus matching technique is strictly protocolized during the customization of patient-specific tinnitus characteristics in reference to their own subjective perception of tinnitus sounds; subsequently, as studies have validated that the therapeutic sound most similar to patient's tinnitus can be beneficial, we believe that our results are in line with our studies [7]. Due to the diversity and complexity of subjective tinnitus sounds, there are some patients whose tinnitus sounds would not match any of the existing edited sound fragments (as in group B) in all 4 steps of the matching test process. These patients often raise negative concerns over whether to receive customized sound therapy, leading them to suspect the curative effect beforehand, and thereby, causing delayed or abrogated sound therapy. However, our study found that the customized sounds, notwithstanding not perfectly matched to the characteristics of patient's tinnitus, still manage to provide therapeutic benefits in the nonmatch group. As such, this suggests that the customized sounds may be prescribed generally to patients with chronic tinnitus.

In this prospective study, we explored and compared the curative effect of the patients with matching and nonmatching tinnitus sounds. The tinnitus sound matching and nonmatching groups both showed a significantly improved effect, as assessed by the scores of HADS-A, HADS-D, THI, and VAS. These results suggested that regardless of the patient's tinnitus sound matching degree, customized sound therapy has a significant efficacy, and the THI score is significantly reduced, which indicates

Relation of Matching Degree and

Therapeutic Assessment in Tinnitus markedly improved tinnitus-induced dysfunction and negative emotional state of the patient. We observed that some patients in the nonmatching group might suspect or even distrust the therapeutic effect before receiving customized sound therapy, owing to their concerns about mismatching of the tinnitus sound. The results of this study provided a positive basis for the promotion of the application of customized sound therapy in clinical diagnosis and treatment of tinnitus patients. Professionals should provide adequate and reasonable pre-education to patients, guiding them to try customized sound therapy and informing them to expect a good therapeutic effect. Furthermore, while receiving treatment, tinnitus patients need to follow the doctor's guidance for compliance in the treatment. In addition, experienced doctors or trained staff are required to provide long-term follow-up guidance and adjust the patient's treatment at first hand, in order to obtain the most optimal treatment effect.

The comparison of the difference between the 2 groups showed that the improvement in HADS-A and THI scores of the tinnitus sound matching group was better than that of the tinnitus sound nonmatching group. The data affirmed that such improvement is related to the degree of hearing loss at the tinnitus side of the patient before treatment. It can be extrapolated that the lighter the hearing loss is, the better the improvement will be. In other words, patients with significant hearing loss who received appropriate hearing loss treatments, for example, using appropriate hearing aids prior to the customized sound therapy are expected to obtain better curative effect afterward. Meanwhile, Tylor found that tinnitus can be reduced or eliminated with cochlear electrical stimulationor [24] and the feasibility of cochlear implant for tinnitus suppression [25]. In addition, no significant difference was detected in the improvement of HADS-D and VAS scores between the 2 groups, and it was not related to the degree of hearing loss of the patients. VAS score is a subjective tinnitus loudness rating scale. Hence, it reflects that patients may concentrate primarily on their tinnitus loudness when they score their tinnitus loudness. Since the customized sound therapy cannot directly reduce the patients' tinnitus loudness, the difference is that patients cannot ignore their tinnitus before treatment while acclimatizing to and ignoring their tinnitus after treatment. Nonetheless, the insignificant difference of HADS-D scores presumably results from the negative emotions related to the distrust/suspicion of our treatment strategy of participants who did not meet the matching requirements. In addition, negative emotion plays a pivotal role in provoking negative physiological and psy-

ORL 2022;84:229-237 
chological changes to human body while simultaneously impacts on the tinnitus per se, thereby forming a continuous vicious feedback loop and further aggravating sensation tinnitus [26]. In such scenario, patients may benefit from establishing a positive mindset or maintain a long-term optimistic and positive attitude when coping with tinnitus. Prescription of antidepressant drugs and consultation to psychiatric professionals can be recommended if patient's negative emotions further worsen during the process of sound therapy, thus, tinnitus treatment could be combined with the psychiatric treatment. Some results also confirmed that other relevant control variables, such as age, gender, and tinnitus duration, have no effect on the customized sound curative effect.

The limitation of this study is the lack of a finer degree of tinnitus sound matching and longer follow-up duration; in addition, this study is not a randomized study. Thus, in future studies, the level of tinnitus sound matching should differentiate in a multiple-level form and longer follow-up duration of patients are warranted to further improve our data. In addition, there remains no objective observation on improving the matching degree of tinnitus patients or idealizing the customized sound, making it more suitable for specific patients. As such, attention should be paid to further expand the soundmatching database, as this study has shown preliminary results that customized sound therapy which is beneficial to tinnitus patients with regard to the therapeutic effect. Furthermore, this study is mainly to study the initial matching degree of patients, in future studies; we will conduct separate studies on the possible changes in tinnitus matching degree during the treatment process.

\section{Conclusions}

Sound therapy is currently an advanced and effective treatment for chronic subjective tinnitus. Customized sound therapy effectively reduces the tinnitus loudness and the negative effects of tinnitus for different individuals; the effect is better than that of broadband noise [27]. This study explored the influence of tinnitus sound matching degree to the efficacy of customized sound therapy, suggesting that customized sound therapy has a significant effect irrespective of patient's tinnitus sound matching degree. Thus, reducing the psychological resistance of patients with nonmatching tinnitus sounds to receive customized sound therapy is critical for improving the curative effect in patients and promoting further clinical application.

\section{Statement of Ethics}

This research complied with the guidelines for human studies and was conducted ethically in accordance with the World Medical Association Declaration of Helsinki. All the subjects (or their parents or guardians) have given their informed consent and signed their consent on the outpatient case file before treatment. This study was approved by the Ethics Review Board. (The name of the Ethics Committee and the reference number: Institutional Review Board of Huadong Hospital Affiliated to Fudan University, 2020K110.)

\section{Conflict of Interest Statement}

The authors have no conflicts of interest to declare.

\section{Funding Source}

This work was funded by the Shanghai Municipal Commission of Science and Technology Fund, China (No. 20Y11902300).

\section{Author Contributions}

J.Y., N.C., and Z.H. designed the research; J.Y. and Z.H. performed the research; J.Y., J.D., J.S., and L.H. followed up the patients and sorted out the data; J.Y., N.C., and Z.H. analyzed the data and wrote the manuscript.

\section{Data Availability Statement}

The data that support the findings of this study are not publicly available due to their containing information that could compromise the privacy of research participants but are available from the corresponding author Zhao Han under reasonable requests.

\footnotetext{
References

1 Møller AR, Langguth B, DeRidder D, Kleinjung T, editors. Textbook of tinnitus. New York: Springer; 2011.

2 Tyler RS, Gogel SA, Gehringer AK. Tinnitus activities treatment. Prog Brain Res. 2007 Feb; 166:425-34.

3 Okamoto H, Stracke H, Stoll W, Pantev C. Listening to tailor-made notched music reduces tinnitus loudness and tinnitus-related auditory cortex activity. Proc Natl Acad Sci U S A. 2010 Jan;107(3):1207-10.

4 Zhang W, Peng Z, Yu S, Song QL, Qu TF, Liu $\mathrm{K}$, et al. Exposure to sodium salicylate disrupts VGLUT3 expression in cochlear inner hair cells and contributes to tinnitus. Physiol Res. 2020 Feb;69(1):181-90.
} 
5 Andersson G, Kaldo-Sandström V, Ström L, Strömgren T. Internet administration of the Hospital Anxiety and Depression Scale in a sample of tinnitus patients. J Psychosom Res. 2003 Sep;55(3):259-62.

6 Hobson J, Chisholm E, El Refaie A. Sound therapy (masking) in the management of tinnitus in adults. Cochrane Database Syst Rev. 2012 Nov;11(11):CD006371.

7 Henry JA, Meikle MB. Psychoacoustic measures of tinnitus. J Am Acad Audiol. 2000 Mar;11(3):138-55.

8 Pan T, Tyler RS, Ji H, Coelho C, Gehringer AK, Gogel SA. The relationship between tinnitus pitch and the audiogram. Int J Audiol. 2009 May;48(5):277-94.

9 Tyler RS, Baker LJ. Difficulties experienced by tinnitus sufferers. J Speech Hear Disord. 1983 May;48(2):150-4.

10 Dauman R, Tyler RS. Some considerations on the classification of tinnitus. In: Dauman R, Tyler RS, editors. Proceedings of the fourth international tinnitus seminar. Amsterdam: Kugler \& Ghedini Publications; 1992. p. 2259.

11 He D, Shou Z, Han Z. [Analysis of the effect and influencing factors of customized sound on tinnitus]. Lin Chung Er Bi Yan Hou Tou Jing Wai Ke Za Zhi. 2019 Jun;33(6):525-8.

12 Tyler R, Ji H, Perreau A, Witt S, Noble W, Coelho C. Development and validation of the tinnitus primary function questionnaire. Am J Audiol. 2014 Sep;23(3):260-72.
13 Knipper M, Zimmermann U, Müller M. Molecular aspects of tinnitus. Hear Res. $2010 \mathrm{Jul}$; 266(1-2):60-9.

14 Tyler RS, Aran JM, Dauman R. Recent advances in tinnitus. Am J Audiol. 1992 Nov 1; 1(4):36-44

15 Yang H, Zheng Y, Zhang Z, Lan J, Chen S, Liang X, et al. [Analysis and comparison of the masking and TRT for patients with subjective tinnitus]. Lin Chung Er Bi Yan Hou Tou Jing Wai Ke Za Zhi. 2010 Sept;24(10): 442-6.

16 Tyler RS, Noble W, Coelho CB, Ji H. Tinnitus retraining therapy: mixing point and total masking are equally effective. Ear Hear. 2012 Sep-Oct;33(5):588-94

17 Hu YQ, Gao MH, Li CJ, Li ZW, Kuang SJ, Zou F. The discussion of therapeutic method and curative effect on chronic subjectivity tinnitus. Chongqing Med. 2014 Nov;43(33):44623.

18 Tyler RS, Perreau A, Powers T, Watts A, Owen R, Ji H, et al. Tinnitus sound therapy trial shows effectiveness for those with tinnitus. J Am Acad Audiol. 2020 Jan;31(1):6-16.

19 Li SA, Bao L, Chrostowski M. Investigating the effects of a personalized, spectrally altered music-based sound therapy on treating tinnitus: a blinded, randomized controlled trial. Audiol Neurootol. 2016 Nov;21(5):296-304.

20 Mahboubi H, Ziai K, Djalilian HR. Customized web-based sound therapy for tinnitus. Int Tinnitus J. 2012 Jan;17(1):26-30.
21 Haab L. Numerical modeling and experimental validation of involuntary attentional capture and long-term habituation by the example of tinnitus aurium [doctoral dissertation]. Homburg: Universität des Saarlandes; 2015.

22 Ma LR, Fu T, Yang YM. Matching test for the therapeutic evaluation of tinnitus. Chin J Otorhinolaryngol Integr Tradit West Med. 2003 Jan;11(1):11-2.

23 Hébert S. Individual reliability of the standard clinical method vs patient-centered tinnitus likeness rating for assessment of tinnitus pitch and loudness matching. JAMA Otolaryngol Head Neck Surg. 2018 Dec;144(12):1136-44.

24 Tyler RS, Rubinstein J, Pan T, Chang SA, Gogel SA, Gehringer A, et al. Electrical stimulation of the cochlea to reduce tinnitus. Semin Hear. 2008 Nov;29(4):326-32.

25 Perreau A, Tyler R, Mancini PC. Programming a cochlear implant for tinnitus suppression. J Am Acad Audiol. 2020 Apr;31(4):3028.

26 Song JJ, Punte AK, De Ridder D, Vanneste S, Van de Heyning P. Neural substrates predicting improvement of tinnitus after cochlear implantation in patients with single-sided deafness. Hear Res. 2013 May;299:1-9.

27 Mahboubi H, Haidar YM, Kiumehr S, Ziai K, Djalilian HR. Customized versus noncustomized sound therapy for treatment of tinnitus: a randomized crossover clinical trial. Ann Otol Rhinol Laryngol. 2017 Oct;126(10): 681-7. 